\title{
Isomorphism and matrix representation of point groups
}

\author{
Wan Heng Fong*, Aqilahfarhana Abdul Rahman, Nor Haniza Sarmin \\ a Department of Mathematical Sciences, Faculty of Science, Universiti Teknologi Malaysia, 81310 UTM Johor Bahru, Malaysia \\ *Corresponding author: fwh@utm.my
}

\section{Article history}

Received 27 February 2018

Revised 28 March 2018

Accepted 21 May 2018

Published Online

\begin{abstract}
In chemistry, point group is a type of group used to describe the symmetry of molecules. It is a collection of symmetry elements controlled by a form or shape which all go through one point in space, which consists of all symmetry operations that are possible for every molecule. Next, a set of number or matrices which assigns to the elements of a group and represents the multiplication of the elements is said to constitute representation of a group. Here, each individual matrix is called a representative that corresponds to the symmetry operations of point groups, and the complete set of matrices is called a matrix representation of the group. This research was aimed to relate the symmetry in point groups with group theory in mathematics using the concept of isomorphism, where elements of point groups and groups were mapped such that the isomorphism properties were fulfilled. Then, matrix representations of point groups were found based on the multiplication table where symmetry operations were represented by matrices. From this research, point groups of order less than eight were shown to be isomorphic with groups in group theory. In addition, the matrix representation corresponding to the symmetry operations of these point groups wasis presented. This research would hence connect the field of mathematics and chemistry, where the relation between groups in group theory and point groups in chemistry were shown.
\end{abstract}

Keywords: Group; isomorphism; matrix representation; point group

\section{INTRODUCTION}

Group theory is a study about an algebraic structure known as group in mathematics and abstract algebra. It is also a mathematical branch in which symmetry of molecules can be determined. Besides being the systematic treatment of symmetry, it is a powerful tool that simplifies the process of obtaining information about molecules. Both group theory and symmetry provide a formal method for identifying the geometry of objects by describing the shapes in their structure.

Point group is an important property of molecules that is widely used in some branches of chemistry, which are crystallography, spectroscopy and quantum chemistry. Besides that, point groups are also used in solid state physics, in which the definitions of all symmetry operations, the multiplication table, all classes, their permutation counterparts and matrix representations, abelian subgroups and also some other subgroups of cubic point group $O_{h}$ have been discussed (Delibas, Aykan, Turkkan, \& Akkus, 2013). A point group can be used to classify the shapes of molecules based on their symmetry elements. It is known as point group because all symmetry elements which include planes, lines and points are intersected at a one point. The notation used to represent point group of a molecule is the Schoenflies notation which is commonly used by spectroscopists and chemists.

The mathematical equivalence between two or more groups is known as isomorphism. It is a map that preserves sets and relations among elements. The concept of isomorphism is beneficial as it reduces the number of groups that need to be studied for obtaining properties of groups. The isomorphic groups have the same form or structure of Cayley table although they are different in notation or in the nature of their elements. Fong (2004) determined the isomorphism of groups of order eight with certain point groups. Hence, the point groups in chemistry and groups in group theory can be mapped using isomorphism.

For each of the point groups, the symmetry operations in a group can be represented by matrices. The concept of matrices in algebra can be applied to the elements in point groups. A set of number or matrices which is assigned to the elements of a group and represented the multiplication of the elements is said to constitute representation of a group (Ferraro \& Ziomek, 1969). Here, each individual matrix is called a representative that corresponded to the symmetry operations of point groups, and the complete set of matrices is called a matrix representation of the group.

This paper was organized as following: Section 1 woud introduce the background of the research. In Section 2, some necessary definitions and notations used in this research were presented. Next, the isomorphisms between point groups of order less than eight with groups in group theory and the matrix representation of point groups of order less than eight were discussed and shown in Section 3. In Section 4 , the results on the isomorphisms and matrix representation of point groups of order less than eight were given. 
Table 1 The List of point groups of order less than eight.

\begin{tabular}{|c|c|c|c|c|}
\hline & Point Group & Symmetry Element & Order & Example Of Molecule \\
\hline \multirow[t]{3}{*}{$\begin{array}{l}\text { The non-axial } \\
\text { groups }\end{array}$} & $C_{1}$ & $E$ & 1 & $\begin{array}{l}\text { Bromochlorofluoromethane, } \\
\text { CHFCIBr }\end{array}$ \\
\hline & $C_{s}$ & $E, \sigma_{h}$ & 2 & $\begin{array}{l}\text { 1-bromo-2-chloroethene, } \\
\mathrm{C}_{2} \mathrm{H}_{2} \mathrm{BrCI}\end{array}$ \\
\hline & $C_{i}$ & $E, i$ & 2 & $\begin{array}{l}\text { 1,2-dichloro-1,2-difluoroethane, } \\
\qquad \mathrm{CHCI}_{2}\end{array}$ \\
\hline \multirow[t]{12}{*}{ The cyclic groups } & $C_{2}$ & $E, C_{2}^{1}$ & 2 & Hydrogen peroxide, $\mathrm{H}_{2} \mathrm{O}_{2}$ \\
\hline & $C_{3}$ & $E, C_{3}^{1}, C_{3}^{2}$ & 3 & Triphenylphosphine, $\mathrm{P}\left(\mathrm{C}_{6} \mathrm{H}_{5}\right)_{3}$ \\
\hline & $C_{4}$ & $E, C_{4}^{1}, C_{4}^{2}=C_{2}, C_{4}^{3}$ & 4 & Tetra-aza copper (II) \\
\hline & $C_{5}$ & $E, C_{5}^{1}, C_{5}^{2}, C_{5}^{3}, C_{5}^{4}$ & 5 & {$\left[\mathrm{Fe}\left(\mathrm{Me}_{5}-\mathrm{Cp}\right)\left(\mathrm{P}_{5}\right)\right]$} \\
\hline & & $E, C_{6}^{1}, C_{6}^{2}=C_{3}^{1}, C_{6}^{3}=C_{2}$, & & \\
\hline & $C_{6}$ & $C_{6}^{4}=C_{3}^{2}, C_{6}^{5}$ & 6 & Alpha-cyclodextrin, $\mathrm{C}_{36} \mathrm{H}_{60} \mathrm{O}_{30}$ \\
\hline & $C_{7}$ & $E, C_{7}{ }^{1}, C_{7}{ }^{2}, C_{7}^{3}, C_{7}^{4}, C_{7}^{5}, C_{7}^{6}$ & 6 & - \\
\hline & $S_{1}$ & $E, \sigma_{h}$ & 2 & $\begin{array}{l}\text { 1-bromo-2-chloroethene, } \\
\mathrm{C}_{2} \mathrm{H}_{2} \mathrm{BrCI}\end{array}$ \\
\hline & $S_{2}$ & $E, S_{2}^{1}=i$ & 2 & $\begin{array}{l}\text { 1,2-dichloro-1,2-difluoroethane, } \\
\mathrm{CHCI}_{2}\end{array}$ \\
\hline & $S_{3}$ & $\begin{array}{c}E, S_{3}{ }^{1}, S_{3}{ }^{2}=C_{3}{ }^{2}, S_{3}^{3}=\sigma_{h} \\
S_{3}{ }^{4}=C_{3}{ }^{1}, S_{3}{ }^{5}\end{array}$ & 6 & Boric acid, $\mathrm{B}(\mathrm{OH})_{3}$ \\
\hline & $S_{4}$ & $E, S_{4}^{1}, S_{4}^{2}=C_{2}, S_{4}^{3}$ & 4 & Tetrabomoneopentane, $\mathrm{C}_{5} \mathrm{H}_{8} \mathrm{Br}_{4}$ \\
\hline & $S_{6}$ & $\begin{array}{c}E, S_{6}^{1}, S_{6}{ }^{2}=C_{3}^{1}, S_{6}^{3}=i \\
S_{6}^{4}=C_{3}{ }^{2}, S_{6}{ }^{5}\end{array}$ & 6 & Ethane, $\mathrm{C}_{2} \mathrm{H}_{6}$ \\
\hline \multirow{6}{*}{$\begin{array}{l}\text { The axial groups } \\
\text { containing mirror } \\
\text { planes }\end{array}$} & $C_{1 h}$ & $E, \sigma_{h}$ & 2 & $\begin{array}{l}\text { Chlorofluoromethane, } \\
\mathrm{CH}_{2} \mathrm{CIF}\end{array}$ \\
\hline & $C_{2 h}$ & $E, C_{2}, S_{2}=i, \sigma_{h}$ & 4 & $\begin{array}{l}\text { 1,2-dichloroethylene (trans), } \\
\qquad \mathrm{C}_{2} \mathrm{H}_{2} \mathrm{CI}_{2}\end{array}$ \\
\hline & $C_{3 h}$ & $\begin{array}{c}E, S_{3}{ }^{1}, S_{3}{ }^{2}=C_{3}{ }^{2}, S_{3}{ }^{3}=\sigma_{h} \\
S_{3}{ }^{4}=C_{3}{ }^{1}, S_{3}{ }^{5}\end{array}$ & 6 & Boric acid, $\mathrm{B}(\mathrm{OH})_{3}$ \\
\hline & $C_{1 v}$ & $E, \sigma_{v}$ & 2 & - \\
\hline & $C_{2 v}$ & $E, C_{2}, \sigma_{v}(x z), \sigma_{v}{ }^{\prime}(y z)$ & 4 & Water, $\mathrm{H}_{2} \mathrm{O}$ \\
\hline & $C_{3 v}$ & $E, C_{3}{ }^{1}, C_{3}{ }^{2}, \sigma_{v}{ }^{\prime}, \sigma_{v}{ }^{\prime \prime}, \sigma_{v}{ }^{\prime \prime}$ & 6 & Ammonia, $\mathrm{NH}_{3}$ \\
\hline \multirow{5}{*}{$\begin{array}{l}\text { The axial groups } \\
\text { with multiple } \\
\text { rotation axes }\end{array}$} & $D_{1}$ & $E, C_{2}^{1}$ & 2 & Hydrogen peroxide, $\mathrm{H}_{2} \mathrm{O}_{2}$ \\
\hline & $D_{2}$ & $E, C_{2}, C_{2}^{\prime}, C_{2}^{\prime \prime}$ & 4 & Twistane, $\mathrm{C}_{10} \mathrm{H}_{16}$ \\
\hline & $D_{3}$ & \multicolumn{2}{|c|}{$E, C_{3}^{1}, C_{3}^{2}, C_{2}(x), C_{2}(y), C_{2}(z)$} & $\begin{array}{l}\text { Ruthenium triethylenediamine, } \\
\qquad \mathrm{Ru}(\text { en })_{3}\end{array}$ \\
\hline & $D_{1 h}$ & $E, C_{2}, \sigma_{v}(x z), \sigma_{v}{ }^{\prime}(y z)$ & 4 & Water, $\mathrm{H}_{2} \mathrm{O}$ \\
\hline & $D_{1 d}$ & $E, C_{2}, S_{2}=i, \sigma_{h}$ & 4 & $\begin{array}{l}\text { 1,2-dichloroethylene (trans), } \\
\qquad \mathrm{C}_{2} \mathrm{H}_{2} \mathrm{CI}_{2}\end{array}$ \\
\hline
\end{tabular}

in the next section, some preliminaries concepts used in this paper were discussed.

\section{LITERATURE REVIEW}

This section included some preliminary concepts and definitions that were used in this paper. first, two basic concepts in point groups that were needed when performing an operation to a molecule, namely symmetry operation and symmetry element, were discussed.

a symmetry operation is a movement of a body where every point of the body is correspondent with an equivalent point (or a similar point) of the body in its own orientation after the movement has been done; while symmetry element is a geometrical element with respect to 
which at least one symmetry operations that might be carried out such as a line, a plane, or a point (cotton, 2008).

Both symmetry operation and symmetry element are interrelated since the operation is defined with respect to the element. There are only four types of symmetry elements and operations to be considered in treating the symmetry of molecules, which are symmetry planes and reflections, inversion center, proper axes and proper rotations, improper axes and improper rotations, denoted as $\sigma, i, C_{n}$ and $S_{n}$ respectively(Cotton, 2008). The identity operation (denoted as $E$ ) which is used to represent any combination of operations which takes the molecule to a same configuration with the original one in treating molecular symmetry since all molecules have the same identity element. Next, the definition of a point group was stated.

\section{Definition 1 (Windle, 1977): Point Group}

A point group is a collection of symmetry elements possessed by a shape or form which all passed through one point in space.

The symmetry of point groups can be classified into six groups, the non-axial groups, the cyclic groups, the axial groups containing mirror plane, the axial groups with multiple rotation axes, the special groups for linear molecules and the cubic groups (Willock, 2009). In this research, the list of point groups was started with point groups of the least symmetry and ended with point groups of order seven in which only four of these six groups were discussed, namely the nonaxial groups, the cyclic groups, the axial groups containing mirror plane and the axial groups with multiple rotation axes. The rest of them which were special groups for linear molecules and the cubic groups were not presented in this research since the lowest order of those groups was infinity and order 12 respectively. Table 1 shows the list of point groups of order less than eight with their respective symmetry elements, order and example of molecules. Next, the equivalence between two or more groups could be found and mapped using isomorphism, in which the definition of isomorphism was stated next.

\section{Definition 2 (Gallian, 2012) : Isomorphism}

An isomorphism $\phi$ from a group $G$ to a group $\bar{G}$ is a one-to-one mapping (or function) from $G$ onto $\bar{G}$ that preserves the group operation, that is $\phi(a b)=\phi(a) \phi(b)$ for all $a, b$ in $G$. If there is an isomorphism from $G$ onto $\bar{G}$, we say that $G$ and $\bar{G}$ are isomorphic, denoted as $\cong$.

In point groups, the stereographic projection is used to visualize the symmetry operations. Stereographic projection or stereogram is a convenient way of mapping the points on the surface of a sphere onto a two-dimensional figure (Gallian, 2012). The notation + is used if the mark is above the plane while the notation o is used if the mark is below the plane.

\section{METHODOLOGY}

In this section, the isomorphism between point groups of order less than eight with groups in group theory and the matrix representation of point groups were determined. Here, the mapping between elements of point groups and groups was shown to fulfill the properties of isomorphism which were-one-to-one, onto and operation preserving. The notation $\cong$ was used to represent the isomorphism.

Here, the isomorphism of the group of order six, $Z_{6}$ was discussed. The group presentation for the group $Z_{6}$ was $\left\langle a \mid a^{6}=e\right\rangle$ where $Z_{6}=\left\{e, a, a^{2}, a^{3}, a^{4}, a^{5}\right\}$. As mentioned earlier, the point group $C_{6}$ consisted of elements $E, C_{6}{ }^{1}, C_{3}{ }^{1}, C_{2}, C_{3}{ }^{2}, C_{6}{ }^{5}$. Fig. 1 shows the stereographic projection for the point group $C_{6}$ (Cracknell, 2016).

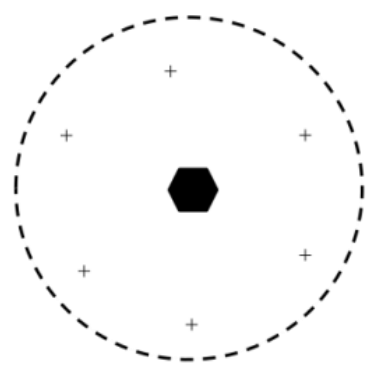

Fig. 1 Stereographic Projection for the Point Group $C_{6}$

The symmetry operations of the point group $C_{6}$ were listed as following:

$E$ : Identity,

$C_{6}{ }^{1}$ : Rotation through $\pi / 3$,

$C_{3}^{1}:$ Rotation through $2 \pi / 3$,

$C_{2}$ : Rotation through $\pi$,

$C_{3}^{2}$ : Rotation through $4 \pi / 3$,

$C_{6}^{5}$ : Rotation through $5 \pi / 3$.

The Cayley table of the point group $C_{6}$ was given in Table 2 .

Table 2 Cayley Table of the Point Group $\boldsymbol{C}_{\mathbf{6}}$.

\begin{tabular}{l|llllll}
\hline$\cdot$ & $E$ & $C_{6}{ }^{1}$ & $C_{3}{ }^{1}$ & $C_{2}$ & $C_{3}{ }^{2}$ & $C_{6}{ }^{5}$ \\
\hline$E$ & $E$ & $C_{6}{ }^{1}$ & $C_{3}{ }^{1}$ & $C_{2}$ & $C_{3}{ }^{2}$ & $C_{6}{ }^{5}$ \\
$C_{6}{ }^{1}$ & $C_{6}{ }^{1}$ & $C_{3}{ }^{1}$ & $C_{2}$ & $C_{3}{ }^{2}$ & $C_{6}{ }^{5}$ & $E$ \\
$C_{3}{ }^{1}$ & $C_{3}{ }^{1}$ & $C_{2}$ & $C_{3}{ }^{2}$ & $C_{6}{ }^{5}$ & $E$ & $C_{6}{ }^{1}$ \\
$C_{2}$ & $C_{2}$ & $C_{3}{ }^{2}$ & $C_{6}{ }^{5}$ & $E$ & $C_{6}{ }^{1}$ & $C_{3}{ }^{1}$ \\
$C_{3}{ }^{2}$ & $C_{3}{ }^{2}$ & $C_{6}{ }^{5}$ & $E$ & $C_{6}{ }^{1}$ & $C_{3}{ }^{1}$ & $C_{2}$ \\
$C_{6}{ }^{5}$ & $C_{6}{ }^{5}$ & $E$ & $C_{6}{ }^{1}$ & $C_{3}{ }^{1}$ & $C_{2}$ & $C_{3}{ }^{2}$ \\
\hline
\end{tabular}

The isomorphism mapping $\phi$ from $Z_{6}$ to the point group $C_{6}$ was given below:

$$
\begin{gathered}
\phi: e \rightarrow E, \\
\phi: a \rightarrow C_{6}{ }^{1}, \\
\phi: a^{2} \rightarrow C_{3}{ }^{1}, \\
\phi: a^{3} \rightarrow C_{2}, \\
\phi: a^{4} \rightarrow C_{3}{ }^{2}, \\
\phi: a^{5} \rightarrow C_{6}{ }^{5} .
\end{gathered}
$$

The mapping wasone-to-one, onto and operation preserving. Below were some calculations used to show the operation preserving between these two groups:

$$
\begin{gathered}
\phi(a a)=\phi\left(a^{2}\right)=C_{3}{ }^{1}, \phi(a) \phi(a)=C_{6}{ }^{1} C_{6}{ }^{1}=C_{3}{ }^{1}, \\
\phi\left(a a^{2}\right)=\phi\left(a^{3}\right)=C_{2}, \phi(a) \phi\left(a^{2}\right)=C_{6}{ }^{1} C_{3}{ }^{1}=C_{2}, \\
\phi\left(a a^{3}\right)=\phi\left(a^{4}\right)=C_{3}{ }^{2}, \phi(a) \phi\left(a^{3}\right)=C_{6}{ }^{1} C_{2}=C_{3}{ }^{2}, \\
\phi\left(a a^{4}\right)=\phi\left(a^{5}\right)=C_{6}{ }^{5}, \phi(a) \phi\left(a^{4}\right)=C_{6}{ }^{1} C_{3}{ }^{2}=C_{6}{ }^{5}, \\
\phi\left(a a^{5}\right)=\phi\left(a^{6}\right)=\phi(e)=E, \phi(a) \phi\left(a^{5}\right)=C_{6}{ }^{1} C_{6}{ }^{5}=E .
\end{gathered}
$$


The rest of the calculations could be obtained in a similar manner. As a conclusion, the group $Z_{6}$ was isomorphic to the point group $C_{6}$ . Here it was also found that $Z_{6} \cong$ point group $C_{6} \cong$ point group $S_{6} \cong$ point group $C_{3 h}$. Similar steps could be applied to the rest of the groups.

Next, the point group was represented in the form of matrices by using the Cayley table of point groups. The matrix representation of the point group of order six, $C_{6}$ was discussed in this section and the Cayley table of this point group has been shown in Table 2.

Firstly for $C_{6}$, using the equation $r_{E}=D(E) r$, where wasis a matrix used to transform $r$, which represented the orginal position of symmetry operations into $r_{E}$, which represented the position of symmetry operations after the operation of performed, then the transformation matrix of $E$ could be represented as

$$
\left(\begin{array}{l}
E \\
C_{6}{ }^{5} \\
C_{3}{ }^{2} \\
C_{2} \\
C_{3}{ }^{1} \\
C_{6}{ }^{1}
\end{array}\right)=\left(\begin{array}{llllll}
1 & 0 & 0 & 0 & 0 & 0 \\
0 & 0 & 0 & 0 & 0 & 1 \\
0 & 0 & 0 & 0 & 1 & 0 \\
0 & 0 & 0 & 1 & 0 & 0 \\
0 & 0 & 1 & 0 & 0 & 0 \\
0 & 1 & 0 & 0 & 0 & 0
\end{array}\right)\left(\begin{array}{c}
E \\
C_{6}{ }^{1} \\
C_{3}{ }^{1} \\
C_{2} \\
C_{3}{ }^{2} \\
C_{6}{ }^{5}
\end{array}\right) .
$$

The procedure could be repeated for each symmetry operation. So, the transformation matrix of $C_{6}^{1}, C_{3}^{1}, C_{2}, C_{3}^{2}$ and $C_{6}^{5}$ be represented in the following:

$$
\begin{aligned}
& \left(\begin{array}{c}
C_{6}{ }^{1} \\
E \\
C_{6}{ }^{5} \\
C_{3}{ }^{2} \\
C_{2} \\
C_{3}{ }^{1}
\end{array}\right)=\left(\begin{array}{llllll}
0 & 1 & 0 & 0 & 0 & 0 \\
1 & 0 & 0 & 0 & 0 & 0 \\
0 & 0 & 0 & 0 & 0 & 1 \\
0 & 0 & 0 & 0 & 1 & 0 \\
0 & 0 & 0 & 1 & 0 & 0 \\
0 & 0 & 1 & 0 & 0 & 0
\end{array}\right)\left(\begin{array}{c}
E \\
C_{6}{ }^{1} \\
C_{3}{ }^{1} \\
C_{2} \\
C_{3}{ }^{2} \\
C_{6}{ }^{5}
\end{array}\right), \\
& \left(\begin{array}{l}
C_{3}^{1} \\
C_{6}{ }^{1} \\
E \\
C_{6}{ }^{5} \\
C_{3}^{2} \\
C_{2}
\end{array}\right)=\left(\begin{array}{llllll}
0 & 0 & 1 & 0 & 0 & 0 \\
0 & 1 & 0 & 0 & 0 & 0 \\
1 & 0 & 0 & 0 & 0 & 0 \\
0 & 0 & 0 & 0 & 0 & 1 \\
0 & 0 & 0 & 0 & 1 & 0 \\
0 & 0 & 0 & 1 & 0 & 0
\end{array}\right)\left(\begin{array}{c}
E \\
C_{6}{ }^{1} \\
C_{3}{ }^{1} \\
C_{2} \\
C_{3}{ }^{2} \\
C_{6}{ }^{5}
\end{array}\right),
\end{aligned}
$$

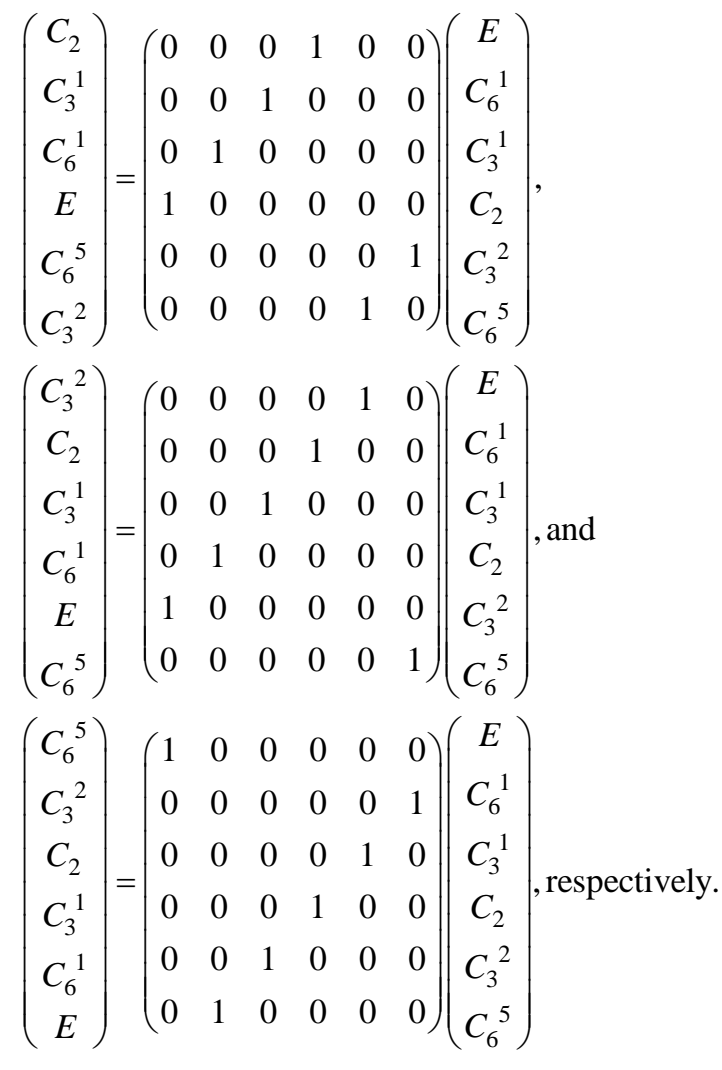

Since the point groups $C_{6}, S_{6}$ and $C_{3 h}=S_{3}$ shared the same structure of Cayley table but were different on the symmetry operations, hence their transformation matrices were the same.

In the next section, the results for the isomorphisms of point groups of order less than eight with groups and the matrix representation of point groups of order less than eight were discussed.

\section{RESULTS AND FINDINGS}

In this research, the isomorphisms between point groups of order less than eight with groups in group theory were found. The group of order one, $Z_{1}$ was isomorphic to the point group $C_{1}$. For group of order two, $Z_{2}$ was isomorphic to all point groups of order two which were $C_{1 h}=C_{s}=C_{1 v}=S_{1}, S_{2}=C_{i}$ and $C_{2}=D_{1}$. Besides that, the group of order three, $Z_{3}$ was isomorphic to the point group $C_{3}$. Next, there were two groups of order four, namely $Z_{4}$ and $Z_{2} \times Z_{2}$. The group $Z_{4}$ was isomorphic to the point groups $C_{4}$ and $S_{4}$; while the group $Z_{2} \times Z_{2}$ was isomorphic to the point groups $C_{2 h}=D_{1 d}, C_{2 v}=D_{1 h}$ and $D_{2}$. The group of order five, $Z_{5}$ was isomorphic to the point group $C_{5}$. Next, there were five point groups of order six, namely $C_{6}, S_{6}, C_{3 h}=S_{3}, C_{3 v}$ and $D_{3}$; while there were only two groups of order six in group theory, namely $Z_{6}$ and $S_{3}$. The group $Z_{6}$ was is isomorphic to the point groups $C_{6}, S_{6}$ and $C_{3 h}=S_{3}$; while the group $S_{3}$ was isomorphic to the point groups $C_{3 v}$ and $D_{3}$. Lastly, the group of order seven, $Z_{7}$ was isomorphic to the point group $C_{7}$. The list of isomorphisms was presented in Table 3. 
Table 3 The isomorphism between point groups and groups.

\begin{tabular}{cccc}
\hline No. & Group & Order & Isomorphic Point Groups \\
\hline 1. & $Z_{1}$ & 1 & $C_{1}$ \\
2. & $Z_{2}$ & 2 & $C_{1 h}=C_{s}=C_{1 v}=S_{1}, S_{2}=C_{i}$, \\
& & & $C_{2}=D_{1}$ \\
3. & $Z_{3}$ & 3 & $C_{3}$ \\
4. & $Z_{4}$ & 4 & $C_{4}, S_{4}$ \\
5. & $Z_{2} \times Z_{2}$ & 4 & $C_{2 h}=D_{1 d}, C_{2 v}=D_{1 h}, D_{2}$ \\
6. & $Z_{5}$ & 5 & $C_{5}$ \\
7. & $S_{3}$ & 6 & $C_{3 v}, D_{3}$ \\
8. & $Z_{6}$ & 6 & $C_{6}, S_{6}, C_{3 h}=S_{3}$ \\
9. & $Z_{7}$ & 7 & $C_{7}$ \\
\hline
\end{tabular}

The concept of isomorphism was beneficial as it helped in reducing the number of groups that needed to be studied in obtaining the properties of groups. The isomorphic groups have the same form or structure of Cayley table although they were different in notation or in the nature of their elements.

Next, the matrix representation of all point groups of order less than eight were obtained by using matrices to represent the elements of the point groups. The representation of each element in point groups was obtained from the Cayley table. For point groups of order one, three, five and seven, each of them have their own matrix representation. All point groups of order two, namely $C_{1 h}=C_{s}=C_{1 v}=S_{1}, S_{2}=C_{i}$ and $C_{2}=D_{1}$ have the same matrix representations. The point group of order four, $C_{4}$ has the same matrix representation with the point group $S_{4}$; while the point group $C_{2 h}=D_{1 d}$ has the same matrix representation with the point groups $C_{2 v}=D_{1 h}$ and $D_{2}$. The point group of order six, $C_{6}$ has the same matrix representation with the point group $S_{6}$ and $C_{3 h}=S_{3}$; while the point group $C_{3 v}$ has the same matrix representation with the point group $D_{3}$.

\section{CONCLUSION}

In this paper, the concept of isomorphism was used to relate point groups with groups in group theory. The mappings between elements in point groups and groups in group theory fulfilled the properties of isomorphism which were one-to-one, onto and operation preserving. In order to visualize the symmetry operations of point groups, stereographic projection was used in this research. Therefore, the isomorphisms of point groups of order less than eight with groups were obtained. Next, matrix representation of point groups of order less than eight was determined. Matrix representation was one of the ways to understand the symmetry operations of a molecule by representing the set of matrices that reflected the group multiplication tables. The symmetry operations were represented by matrices since matrices could be used in dealing with transformation coordinates. In this paper, the matrix representation of all point groups of order less than eight was obtained from the Cayley table.

\section{ACKNOWLEDGEMENT}

The first and third author would like to thank the Ministry of Higher Education (MOHE) Malaysia and Research Management Center (RMC), UTM for the financial funding through Research University Grant Vote No. 13H18. The second author would also like to thank MOHE for the financial funding through MyBrain15 scholarship.

\section{REFERENCES}

Cotton, F. A. 2008. Chemical Applications of Group Theory. John Wiley \& Sons.

Cracknell, A. P. 2016. Applied group theory: The commonwealth and international library: Selected readings in physics: Elsevier.

Delibas, A., Aykan, V., Turkkan, D., Akkus, H. 2013. Point groups in solid state physics i: Point group oh. American Journal of Modern Physics, 2, 2, 81-87.

Ferraro, J. R., Ziomek, J. S. 1969. Introductory group theory; and its application to molecular structures: Plenum Press.

Fong, W. H. 2005. Some Characterizations of Groups of Order 8. (Master Dissertation), Universiti Teknologi Malaysia, Malaysia.

Gallian, J. 2012. Contemporary Abstract Algebra: Nelson Education.

Willock, D. 2009. Molecular Symmetry: John Wiley \& Sons.

Windle, A. H. 1977. A First Course in Crystallography: G. Bell. 